\title{
Iluminación natural en aulas: análisis predictivo dinámico del rendimiento lumínico-energético en clima soleados
}

\author{
Natural daylighting in classrooms: predictive and dynamic \\ analysis of the luminous and energy consumption \\ performance in sunny weather
}

\section{Juan Manuel Monteoliva Andrea Pattini}

\section{Resumen

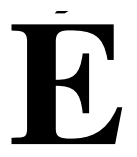

n ciudades como Mendoza (Argentina), donde predomina el cielo claro con sol, la radiación solar es lo suficientemente energética en términos de eficacia luminosa. Esto permite minimizar, y en algunos casos prescindir, de la energía eléctrica consumida por los espacios interiores durante las horas diurnas. El objetivo del presente trabajo es analizar y comparar la autonomía de la iluminación natural y el consumo eléctrico en aulas representativas, tradicionales y bioclimáticas, construidas en la ciudad de Mendoza y el impacto de diferentes husos horarios. La metodología empleada fue: relevamiento físico y fotométrico de aulas reales, configuración de archivos climáticos, y simulaciones y análisis de métricas dinámicas de iluminación natural y consumos eléctricos para iluminación. Los resultados evidenciaron: mayor autonomía de la iluminación natural (15\%) en aulas bioclimáticas; asimismo un aporte adicional del 3\% a partir del uso horario UTC-4 (actualmente no vigente en Argentina, siendo el oficial el UTC-3); y un ahorro del 30\% con la utilización de nuevas tecnologías de control de encendido-apagado en la iluminación artificial. Los resultados obtenidos esperan contribuir a una mayor concientización sobre los beneficios de los edificios escolares energéticamente eficientes; como así también la importancia de la iluminación natural como factor influyente en la energía, salud y confort.

Palabras claves: Iluminación natural. Consumo eléctrico. Escuelas.

\section{Abstract}

In towns like Mendoza (Argentina), where sunny, clear skies predominate, solar radiation provides high values of luminous efficacy. This allows minimizing, and sometimes eliminating, the consumption of electricity in indoor spaces during daylight hours. The goal of this study is to analyze and compare the autonomy of daylight and electricity consumption in classrooms, traditionally built and bioclimatic, in the city of Mendoza. The methodology was divided into the

Juan Manuel Monteoliva Laboratorio de Ambiente Humano y Vivienda San Martín - Mendoza - Argentina

Andrea Pattini Laboratorio de Ambiente Humano y Vivienda Mendoza - Argentina

Recebido em 05/07/13 Aceito em 18/11/13 following stages: physical and photometric survey of real classrooms, weather data settings, and simulation and analysis of dynamic daylight metrics and electricity consumption for lighting. The results clearly showed increased autonomy of daylighting (15\%) in spaces with bioclimatic design compared with traditional classrooms; an additional $3 \%$ by using time zone UTC-4 (currently not applicable in Argentina, as the official one is UTC-3); and, finally, 30\% savings through using new technologies for the on/off control of artificial lighting. These results will hopefully contribute to a greater awareness of the benefits of energyefficient school buildings, as well of the importance of daylighting as a factor in energy savings, health and comfort.

Keywords: Daylight. Electricity consumption. School. 


\section{Introducción}

La iluminación juega un papel fundamental para el desarrollo de las actividades sociales, educativas, comerciales e industriales. Si bien la tecnología ha evolucionado en el campo de la iluminación artificial - generando una mayor eficiencia energética en las luminarias - aún es posible minimizar y en algunos casos prescindir de la energía eléctrica consumida por ésta, durante las horas diurnas. En ciudades donde predomina el cielo claro con sol, como el caso de Mendoza (Argentina) con un promedio anual de duración de sol de 2850 horas, la radiación solar es lo suficientemente "energética" en términos de eficacia luminosa ${ }^{1}$. Esto promueve la cantidad y calidad adecuada de la luz natural en los espacios interiores para realizar tareas visuales diurnas en confort, con un ahorro energético de varias horas al día (PATTINI, 2009), equivalente al 50-80\% del consumo energético requerido por la iluminación artificial (BODART; DE HERDE, 2002). Aspectos importantes, sobre todo, en períodos de crisis energética como la que vive actualmente la región.

Sin embargo, el uso de la iluminación natural como fuente dinámica iluminante, requiere cuidadosa planificación en su diseño. Éste, no sólo deberá contemplar los altos niveles de iluminación - requeridos por las tareas visuales - sino también reducir la luz solar directa y los altos brillos y contrastes como posibles causantes de un acondicionamiento lumínico no adecuado del espacio. De no ser así, la luz solar disponible, será eliminada por falta de confort térmico-lumínico. Es decir, se anulará el aporte de luz natural y generará espacios sombríos (perdiendo la disponibilidad de luz natural característica de la región); consumiendo así mayor energía eléctrica en iluminación artificial. La falta de control de la luz solar en un espacio, propende al diseño de un hábitat energéticamente no sustentable, transformando al sector edilicio no residencial en un gran consumidor de energía eléctrica (PATTINI, 2009). Por lo tanto, la decisión más crítica para conocer el comportamiento de la iluminación natural en los interiores de los edificios se encuentra durante la fase del diseño. Esto genera paralelamente la necesidad de contar con nuevas metodologías que permitan la evaluación del factor iluminación natural desde la propia naturaleza dinámica de la fuente natural.

Asimismo, existen otras medidas para obtener un ahorro de energía eléctrica y un mejor aprovechamiento de la disponibilidad de luz natural; y es a partir de una adecuada selección del huso horario de la región coincidente con la hora solar. Así como el empleo de estrategias de diseño a través de sistemas innovativos de iluminación natural. A partir de lo mencionado, se plantea el siguiente trabajo con el objetivo de analizar y comparar dinámicamente la autonomía de la iluminación natural en diferentes tipologías de aulas representativas y construidas de la ciudad de Mendoza en función de su diseño tradicional o bioclimático. Este nuevo paradigma dinámico aporta nuevas métricas al análisis predictivo anual del factor de iluminación natural. La importancia de combinar el uso de espacios reales con modelos predictivos de precisión - simulaciones - radica en un mejor entendimiento de la problemática abordada. Es decir, este tipo de estudios permiten una mayor validación de los resultados obtenidos espacios reales; y un mayor entendimiento de los resultados predictivos - simulaciones. Cabe destacar que este trabajo forma parte de una tesis doctoral en proceso denominada: "Iluminación en aulas. La dinámica de la luz natural en cielos claros y su incidencia en el rendimiento atencional de los alumnos [...]" (MONTEOLIVA, 2010).

Los criterios de selección de los casos de estudios, fueron: tipologías de aulas construidas pertenecientes a escuelas públicas características de la región:

(a) sin estrategias bioclimáticas, o bien tradicionales; $y$

(b) con estrategias de diseño bioclimático.

A partir de ello, se analizará las autonomías de la iluminación natural en cada tipología y el impacto del huso horario vigente GMT-3 $\left(-45^{\circ}\right)$ y los correspondientes a su ubicación geográfica GMT-4 $\left(-60^{\circ}\right)$ y GMT-5 $\left(-75^{\circ}\right)$ (Figura 1$)$. Como así también, el consumo eléctrico de iluminación artificial mediante el uso de sistemas de control manual y por fotosensor y dimerizado.

\section{La Argentina y los husos horarios}

La Argentina se encuentra junto a Chile y Uruguay en el cono sur del continente americano, ubicada en su totalidad en las franjas de husos horario GMT-4 $\left(-60^{\circ}\right)$ y GMT-5 $\left(-75^{\circ}\right)$. Sin embargo, el horario oficial actualmente empleado es el GMT-3 $\left(-45^{\circ}\right)$ alejado de la hora solar.

\footnotetext{
${ }^{1}$ Eficacia luminosa: cociente entre la iluminancia e irradiancia y sus valores dependerán de la altitud del sol, de la nubosidad y de la concentración de aerosoles y vapor de agua en la atmósfera
} 
Figura 1 - Huso Horario en vigencia y los próximos al territorio nacional

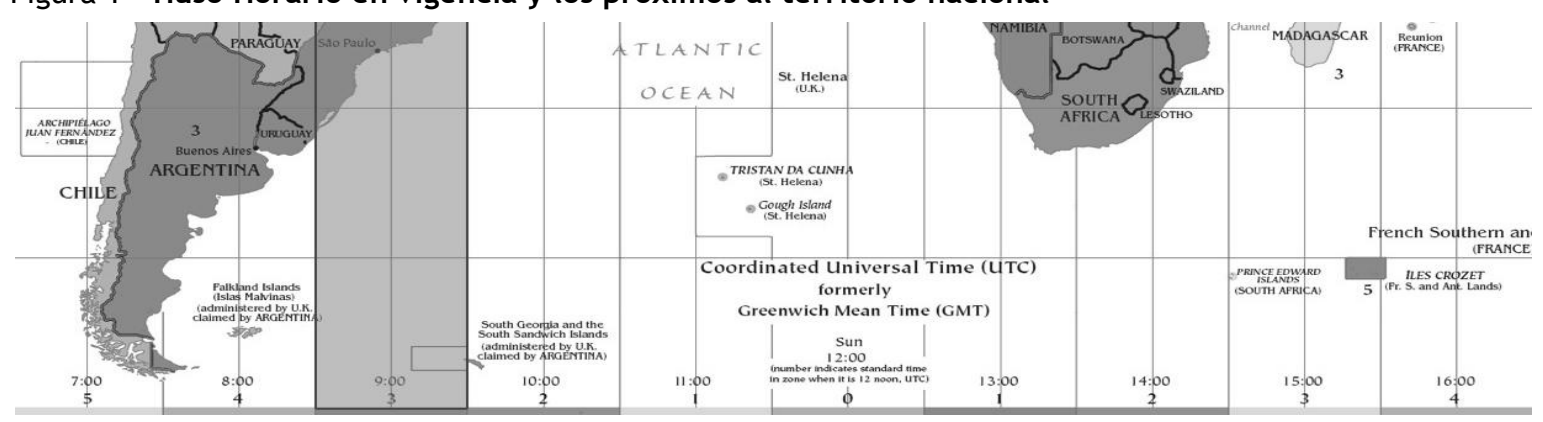

La historia de la Argentina y los husos horarios comienza en 1891 con la primera disposición legal de husos horarios y fue obra de la Municipalidad de Rosario, la cual implementó como hora oficial de la ciudad (GMT-4), correspondiente al Observatorio de Córdoba. En 1894, por decreto del Ejecutivo Nacional, se adopta como horario oficial del país la hora establecida por Rosario GMT-4 manteniéndose vigente hasta 1920 - año que Argentina implementa el sistema internacional unificado de husos horarios (GMT). Diez años después, en 1930, Argentina comienza a adoptar la "hora de verano", adelantando la hora oficial durante el periodo estival para aprovechar mejor la luz solar; la cual siguió implementándose con algunas intermitencias hasta el año 1969. En 1974 se realiza un cambio de horario en verano, partiendo del ya modificado en veranos anteriores que no se había corregido; del GMT-4 a GMT-3. Asimismo durante el período 1988-1992 se cambia nuevamente el horario de verano a GMT-2 partiendo nuevamente del horario no corregido de GMT-3. Finalmente en 1999, la Ley 25.155 estableció al Huso Horario GMT-4, con cambio a GMT-3 desde octubre hasta marzo de cada año. Sin embargo, el Decreto 186/00 prorrogó en el año 2000 la aplicación de dicha Ley, dejando vigente el huso GMT-3 para todo el territorio nacional hasta la fecha sin cambios estacionales. Se considera importante, a partir de esta situación, poder cuantificar el impacto del uso de una hora oficial no coincidente con la hora solar en vistas de promover el uso energético de la luz natural.

\section{La iluminación natural, fuente de luz y algo más}

La iluminación natural en edificios escolares es una de las características físicas más influyentes del aula (PHILLIPS, 1997) y debe ser considerada una variable del ambiente educativo a estudiar en profundidad (DUNN et al., 1985). No sólo, por la oportunidad de ahorros energéticos-ambientales (ya que de la distribución de los consumos eléctricos en edificios escolares, el $66 \%$ del gasto eléctrico se realiza en las aulas) que ésta representa, sino también por la importancia de su ambiente visual. Éste incide en la capacidad de un estudiante para percibir estímulos visuales afectando su actitud mental (TAYLOR; GOUSIE, 1988; EVANS; FERGUSON, 2011) y por lo tanto su rendimiento (HESCHONG, 1999; OKURA, HESCHONG; WRIGHT, 2000). Estudios sobre la iluminación en escuelas sugieren que la luz natural mejora la atención (ISON; PATTINI, 2009), el comportamiento (ANTROP; ROEYERS; DE BAECKE, 2005), los estados de ánimos (VEITCH; GIFFORD; HINE, 1991; VEITCH; NEWSHAM, 1998; TONELLO, 1998) y la sociabilidad del niño. Es decir, la iluminación natural, no sólo sigue siendo un factor predominante en el entorno percibido (WIENOLD; CHRISTOFFERSEN, 2006; ANDERSEN, 2007), el cual en ciertas condiciones es preferido ante la iluminación artificial como fuente de iluminación (GALASIU; VEITCH, 2006; BOYCE; HUNTER; HOWLETT, 2003); sino que produce potenciales beneficios en la salud (REA, 2002; LESESNE; VISSER; WHITE, 2003; WEBB, 2006).

Finalmente, podemos resaltar entonces, la importancia de la iluminación natural en los espacios educativos desde diferentes perspectivas como la energía, salud y confort; y la necesidad de profundizar en los diseños y tecnologías y métodos de cálculos predictivos dinámicos, para un mejor aprovechamiento de la luz natural dentro de estas instituciones. La importancia del ambiente educativo radica, no sólo, en que son espacios en los que la mayoría de los niños juega, aprende, transita e interactúa gran parte de su jornada diaria; sino que es el espacio y tiempo en movimiento, donde los participantes desarrollan capacidades, competencias, habilidades y valores (CENTRO..., 1997). Sin embargo dentro del mundo de la escuela, es el aula donde se ponen en escena las más fieles y verdaderas interacciones, es por ello que muchos investigadores la han denominado " $\mathrm{el}$ momento crucial del acto educativo”. 


\section{Caso de estudio}

Los criterios empleados en la selección de los casos reales de estudio para la ciudad de Mendoza fueron: tipologías de aulas construidas pertenecientes a escuelas públicas características de la región (a) tradicionales y (b) con estrategia de acondicionamiento de energía solar pasiva bioclimática. El objetivo planteado, a partir de estos criterios fue la evaluación y comparación de las autonomías de la iluminación natural en cada tipología y el aprovechamiento energético de las mismas. Las instituciones educativas seleccionadas fueron:

(a) Escuela República de Chile No 1256: esta institución se encuentra emplazada a $3 \mathrm{~km}$ de la capital, provincia de Mendoza (32.52.49 S, 68.52.45 E -853msm-); sitio de densidad edilicia media con obstrucciones parciales a los aventanamientos generadas por edificios cercanos y arbolado (Figura 2). La selección de esta institución educativa, se basó en estudios realizados por el Grupo de Ambiente Humano y Vivienda (LAHV) - INCIHUSA CCT CONICET Mendoza e información complementaria brindada por la Dirección de Ampliación y Construcción de la Subsecretaria de Infraestructura Escolar de la provincia - a través del Arq. Gustavo Colombo -; sobre las principales tipologías de escuelas más representativas del parque edilicio educativo de la provincia de Mendoza (Argentina). La arquitectura general presenta una tipología en bloque de aulas compacto con desarrollo principal este. Este bloque (ala norte simétrico con ala sur) posee seis (6) aulas, cuatro (4) orientas al este y dos al norte, vinculadas por un pasillo de circulación cubierto. Para su estudio, fueron seleccionadas tres tipologías diferentes coexistentes en la institución (A1), (A2) y (A3) (Figura 4); y

(b) Escuela Marcelino H. Blanco No 4042: esta institución se encuentra emplazada en el Departamento de La Paz, provincia de Mendoza (33.27.31 S, 67.32.45 O -509msm-), jurisdicción que dista a $140 \mathrm{~km}$ de la Capital (Figura 3). Este edificio educativo corresponde a uno de los nueve (9) edificios educativos bioclimáticos actuales de la provincia. Éstos fueron diseñados y transferidos a la provincia de Mendoza (Argentina) por el Grupo de Ambiente Humano y Vivienda (LAHV) - INCIHUSA CCT CONICET Mendoza. Para su construcción fueron estudiados, en sus espacios principales, el acondicionamiento con energía solar pasiva (PATTINI, 2009). La arquitectura general presenta una tipología en bloque de aulas compacto con desarrollo principal con orientación norte. Este bloque posee seis (6) aulas: tres (3) alineadas al norte y tres (3) al sur, vinculadas por el pasillo circulación cubierto. Para proveer de ganancia solar norte a ambas tiras de aulas, las tres correspondientes a la tira sur, poseen ventanas altas orientadas al norte por diferencia de techo (MITCHELL et al., 1999). Como caso de estudio, fue seleccionada un aula (A4) perteneciente al bloque de aulas cuya orientación de ventanas de visión directa al exterior eran norte (Figura 5).

Figura 2 - Caso de Estudio - entrada a las instituciones educativas

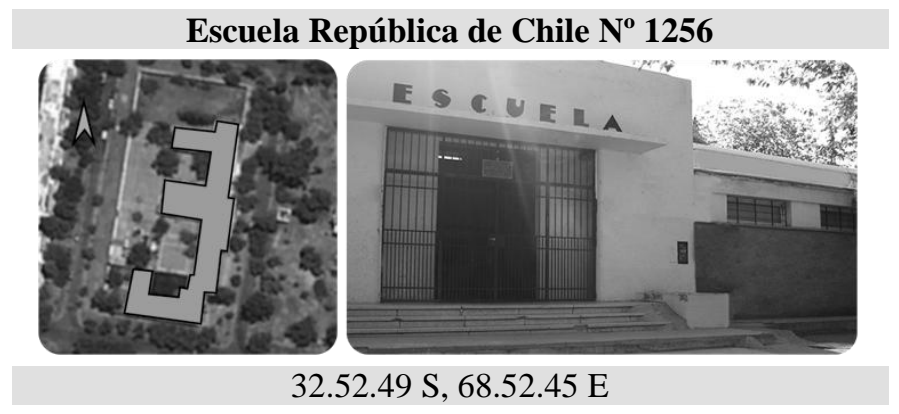

Figura 3 - Vista satelital - entrada a las instituciones educativas

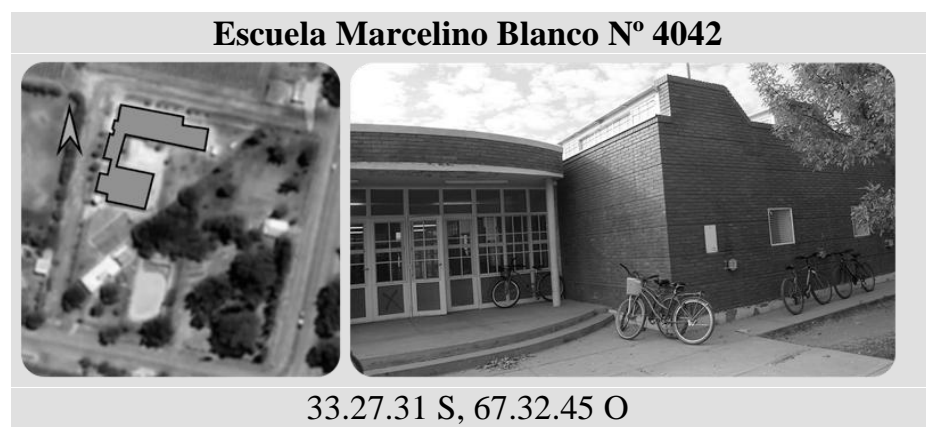

238 Monteoliva, J. M.; Pattini, A. 
Figura 4 - Relevamiento físico del caso de estudio a: (A1), (A2) y (A3); y b: (A4)

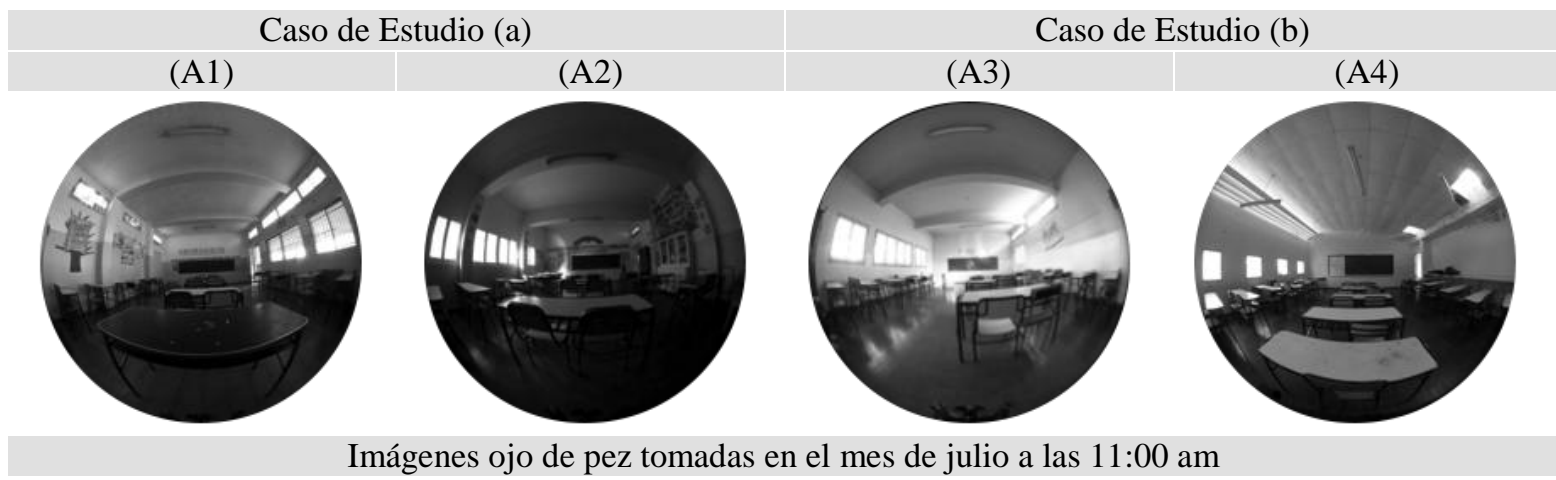

Figura 5 - Relevamiento físico de las tipologías analizadas en los casos de estudio (a) y (b)

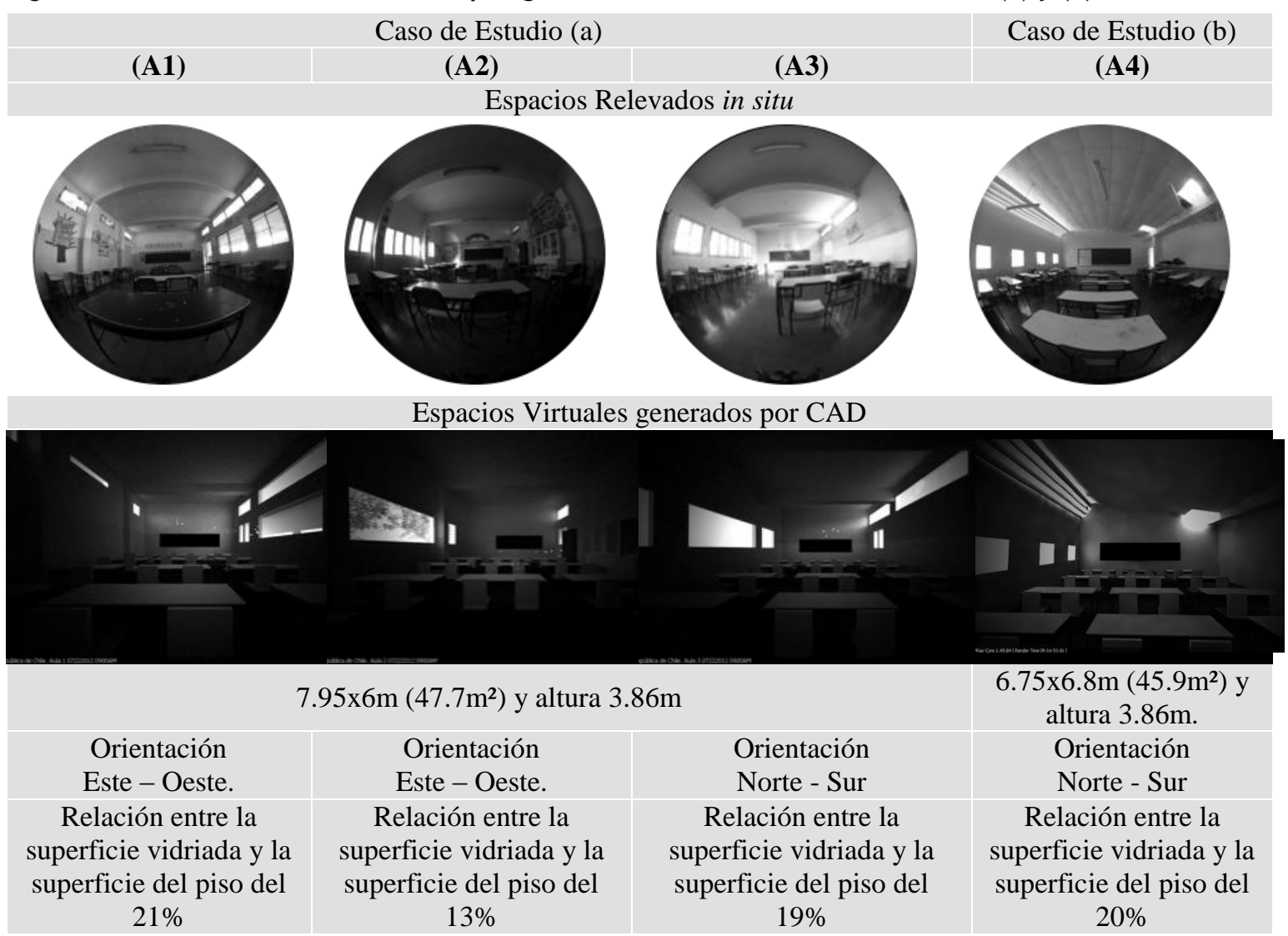

\section{Metodología}

La metodología empleada podemos dividirla en tres etapas principales:

(a) revestimento físico y fotométrico de aulas reales (sistemas de iluminación natural en uso);

(b) configuración de archivos climáticos para los diferentes usos horarios; $y$

(c) simulaciones y análisis de métricas dinámicas de iluminación natural y consumo eléctrico.

\section{Relevamiento físico y fotométrico de aulas reales (sistemas de iluminación natural en uso)}

En el caso de estudio (a) (Figura 4), las aulas seleccionadas (A1), (A2) y (A3) presentan la misma arquitectura de bloque compacto de $7.95 \mathrm{x}$ $6 \times 3.86 \mathrm{~m}\left(47.7 \mathrm{~m}^{2}\right)$ con un sistema de iluminación natural de superficies vidriadas y las siguientes características distintivas:

(a) aula 1 (A1): aula con orientación E-O). La principal superficie vidriada se ubicada en la fachada $(\mathrm{O})$, a la altura de visión, abarcando 
aproximadamente un $90 \%$ del largo la misma. Sin embargo el acceso de la radiación solar actualmente se ve obstaculizado parcialmente por una galería exterior. En relación a las superficies vidriadas de la fachada (E), se ubicadas en la parte superior; sin embargo han sido bloqueadas por los ocupantes en respuesta a la falta de confort que éstas proporcionan. La relación entre la superficie vidriada (SV) y la superficie del piso (SP) es del $21 \%$;

(b) aula 2 (A2): aula con orientación, al igual que (A1), (E-O). La principal superficie vidriada se ubica en la fachada (E), a la altura de visión, abarcando aproximadamente un $90 \%$ del largo la misma. Sin embargo el acceso de la radiación solar actualmente se ve obstaculizado parcialmente por arbolado exterior de especie plátano. Tipología más representativa del arbolado urbano de la ciudad de Mendoza (CANTÓN; DE ROSA; KASPERIDUS, 2003). Si bien, la mayoría de especies presentes en modelo oasis son del tipo caducifolio, el plátano es de follaje perenne, con la particularidad de mantener la hoja durante todo el año, a partir de su condición de marcescente (CÓRICA, 2009). La relación entre (SV) y (SP) es del $13 \%$;

(c) aula 3 (A3): aula con orientación (N-S). El sistema de iluminación natural consta principalmente de una superficie vidriada ubicada en la fachada (S), a la altura de visión, abarcando aproximadamente un $90 \%$ del largo la misma. En relación a los aventanamientos de la fachada $(\mathrm{N})$ se ubica en la parte superior y se encuentra parcialmente obstaculizado por arbolado exterior de especie ciprés. Como toda conífera, son de hojas perenne y presentan un porte piramidal. $\mathrm{La}$ relación entre la superficie vidriada $(\mathrm{SV})$ y la superficie del piso (SP) es del 19\%.

En el caso de estudio (b) (Figura 4), el aula seleccionada (A4) presentan una arquitectura de bloque compacto de $6.80 \times 6.75 \times 3.10 \mathrm{~m}$ (45.9 $\mathrm{m}^{2}$ ) con un sistema de iluminación natural de ventana unilateral y cenital y las siguientes características distintivas el aula tiene orientación (N-S). La principal superficie vidriada se ubica en la fachada $(\mathrm{N})$, en la parte superior abarcando aproximadamente el $90 \%$ del largo de la misma y cuatro ventanas a la altura de la vista. El sistema de iluminación natural del aula se completa con difusores opacos en el interior de las ventanas altas; con acabado en esmalte sintético color blanco (MITCHELL et al., 1999). La relación entre (SV) y (SP) es del $20 \%$.

En cuanto a las características fotométricas, y específicamente la información concerniente a las propiedades ópticas de las superficies, fue obtenida a partir de mediciones in situ. Se determinaron las luminancias de los materiales predominantes en el ambiente (paredes, techos, piso y mobiliario) para caracterizarlos a partir de sus reflectancias en el modelo virtual. Estas mediciones se llevaron a cabo de acuerdo al protocolo de medición de Fontoynont (1999). El instrumental empleado fue un luminancímetro Minolta LS 110 (certificado de calibración, ángulo de lectura de $1 / 3^{\circ}$ y rango de medición de 0.01 a $999.900 \mathrm{~cd} / \mathrm{m}^{2}$ ) y cartillas patrón Kodak.

\section{Configuración de archivos climáticos para los diferentes usos horários}

Actualmente, las herramientas de simulación predictiva pueden modelar con precisión el comportamiento de la iluminación natural en los espacios interiores, disminuyendo la demanda y el empleo de modelos a escala. Sin embargo, estas nuevas metodologías de análisis dinámico requieren nuevos parámetros para la obtención de datos de precisión. Éstos se basan en la correcta descripción y caracterización de la fuente de luz global y directa (PÉREZ; SEALS; MICHALSKY, 1993). Por esta razón, la disponibilidad de datos de la distribución de radiación solar global, resulta fundamentalmente importante. Para este estudio, fue empleada la base climática correspondiente a la ciudad de Mendoza (ARG_MendozaCCT), generada a partir de la información brindada por la estación de medición de iluminación natural del Instituto de Ciencias Humanas Sociales y Ambientales (INCIHUSA) - Laboratorio de Ambiente Humano y Vivienda, ubicado en el Centro Científico y Tecnológico Mendoza (CCT Mendoza) (32.53S y 68.51O) (MONTEOLIVA; VILLALBA; PATTINI, 2012). El modelo de conversión de datos de irradiancias globales horizontales en irradiancia directa empleado fue DISC, producido por el Centro de Datos de Recursos Renovables (RReDC) de Estados Unidos. Esta herramienta (DISC), es un código para el calculo de la radiación solar directa, desarrollado y validado por el Dr. E. Maxwell. Daryl Myers, del Laboratorio Nacional de Energías Renovables (NREL) (MAXWELL, 1987). La conversión de los datos se basa en una relación entre el índice de claridad global (Kt) y el índice de claridad directa normal $(\mathrm{Kn})$, y la geometría solar.

A partir de la base climática ARG_MendozaCCT y la herramienta Weather Manager de Autodesk ECOTECT, se generan los archivos climáticos para diferentes Tiempos Universales Coordinados (UTC) correspondientes al territorio nacional: (UTC-3) - en vigencia; (UTC-4) y (UTC-5). Los archivos climáticos anuales hora-hora generados 
fueron: ARG_MendozaCCT_UTC-3; ARG_MendozaCCT_UTC-4 y ARG_MendozaCCT_UTC5 (latitud -32.5; longitud 68.5 - elevación del sitio $700 \mathrm{msm}$ ) en extensiones (*.wea) y (*.epw) - los términos GMT y UTC, en este contexto, equivalen a mismo concepto. Sin embargo, cabe destacar que la condición generada de UTC-5 fue tan sólo a modo de control (huso horario correspondiente para la región oeste del país, entre ellas la provincia de Mendoza); considerando como huso horario más representativo del territorio nacional UTC-4 - mayor porcentaje de territorio nacional dentro de esta franja horaria. Por lo tanto, el análisis fue basado en las condiciones de UTC-3 actualmente en vigencia - y UTC-4 - más representativo.

\section{Simulaciones y análisis de métricas dinámicas de iluminación natural y consumo eléctrico}

El primer paso en la evaluación de la capacidad visual y la eficiencia energética proporcionada por la luz natural requiere una estimación precisa de la cantidad de luz que ingresa a un edificio (LI, 2004). Es por ello, que la última década se han generado múltiples avances en la forma de analizar numéricamente el comportamiento general de la iluminación natural en los diversos espacios. Entre los principales avances podemos mencionar: no sólo, cálculos predictivos basados en modelos de cielo CIE (CIE Overcast, CIE Clear, CIE Sunny) simulación estática - (JARVIS; DONN, 1997; WARD; SHAKESPEARE, 1998); sino basados en archivos de clima y distribución de luminancia de cielo (PÉREZ; SEAL; MICHALSKY, 1993; REINHART, 2006; REINHART; MARDALJEVIC; ROGERS, 2009) - simulación dinámica. Este nuevo paradigma dinámico aporta nuevas métricas al análisis predictivo anual del factor de iluminación natural (REINHART; MARDALJEVIC; ROGERS, 2006): DF (coeficiente de luz diurna, utilizado para tipo de cielo nublado o uniforme), DA (luz natural autónoma), DAcon (luz natural autónoma ponderada), UDI (iluminación natural útil), DSP (porcentaje de saturación de luz natural) y Exposición de luz natural anual.

En este trabajo en particular, debido a la característica de cielo claro de la región y los objetivos planteados, las métricas dinámicas empleadas fueron DA y DAcon con un valor de 500 [lux] y una grilla de nueve (9) puntos por aula, generada a partir del plano útil a $80 \mathrm{~cm}$ sobre el solado del local - considerado como la zona del plano de trabajo, determinada por las líneas trazadas a $1 \mathrm{mt}$ de las paredes - $\left(\right.$ IRAM $^{2} \mathrm{AADL}^{3}$ J20 02 (INSTITUTO..., 1970)). Cabe destacar que los puntos del grilla fueron calculados con ecuación (Eq. 1) correspondiente al nuevo Protocolo de medición del factor iluminación en puestos de trabajo de la Superintendencia de Riesgos de Trabajo (MINISTERIO..., 2012).

Asimismo la iluminancia mínima de 500lux (sobre superficie de trabajo o pupitre), fue seleccionada a partir del criterio empleado en la normativa nacional vigente para edificios escolares (IRAM AADL J20 04 (INSTITUTO..., 1974)) y los Criterios y Normativa Básica de Arquitectura Escolar (MINISTERIO..., 1998). Cabe destacar, en las métricas dinámicas seleccionadas para el estudio, que mientras (DA500lux) es el porcentaje de tiempo durante el cual se mantiene un nivel mínimo de iluminación en el plano de trabajo; (DAcon500lux) (ROGERS, 2006) otorga créditos parciales a los valores cercanos al valor de consigna requerido. Los parámetros de simulación del ambiente empleados corresponden a una escena simple: (ab) inter-reflexiones 5; (ad) divisiones 1000; (as) muestreo 100; (aa) precisión 0.1; (ar) resolución 300; (dt) umbral directo 0; (ds) sub-muestreo directo $0 \mathrm{y}$ fueron desarrollados en su totalidad en RADIANCE (WARD; SHAKESPEARE, 1998) y DAYSIM (REINHART et al., 2011) integrados en la herramienta DIVA generada para el entorno de Rhinoceros 3D (MCNELL et al., 2010). DIVA es una herramienta de análisis predictivo dinámico del factor de iluminación natural y consumo energético (JACUBIEK; REINHART, 2012). Esta herramienta emplea métodos de optimización del cálculo de iluminancia y distribución de luminancia bajo distintas condiciones climáticas información climática del sitio en estudio - a lo largo del año, bajo el Modelo Pérez de Cielo (PEREZ et al., 1990; PEREZ; SEALS; MICHALSKY, 1993). Así también, fue generado a partir de lo establecido por la Dirección General de Escuelas (MINISTERIO..., 2009), un archivo de ocupación del espacio (schoolyearwithholidays.csv) con la siguiente descripción de uso: lunes a viernes de 8:00 a 18:00 con vacaciones invernales y estivales (Tabla 1).

Puntos del Grillado $=\left(\frac{A \times L}{h m \times(A \times L)}+2\right)^{2} \quad$ Eq. 1 Donde:
(A) es el ancho;
(L) el largo; y

\footnotetext{
${ }^{2}$ Instituto Argentino de Normalización y Certificación (IRAM).

${ }^{3}$ Asociación Argentina de Luminotecnia (ADDL).
} 
$(\mathrm{hm})$ altura de montaje, siendo $(\mathrm{hm})$ la distancia vertical considerada entre la superficie de trabajo y la fuente artificial.

El análisis predictivo del consumo eléctrico fue evaluado por el algoritmo de simulación Lightswitch (REINHART, 2004) integrado a DIVA. Este algoritmo predice, a partir del criterio anual de ocupación del espacio (schoolyearwithholidays.csv) y la iluminancia mínima requerida sobre plano de trabajo (500lux para aulas en estudio), el consumo energético anual $[\mathrm{KWh}]$ de la iluminación artificial complementaria. Los sistemas evaluados de control del apagado y encendido de la iluminación artificial fueron: control manual $(M c)$ y por fotosensor y dimerizado $(D c)$ para las tipologías (A1), (A2), (A3) y (A4) en condiciones de (UTC3) y (UTC-4). Los parámetros empleados en $(M c)$ fueron: potencia de total de los artefactos $500 \mathrm{~W}$ (equivalente al sistema de iluminación artificial complementaria existente en aulas evaluadas de 6 artefactos de 2 tubos fluorescentes T8 de 36W cada uno, sin pantalla difusora); y en (Dc) operación fotosensor y dimerizado; potencia de total de los artefactos $500 \mathrm{~W}$; iluminancia requerida 500lux; factor de pérdida en el balasto $12 \%$ y cantidad de energía perdida durante período de apagado - especial en sistemas automáticos - 15.0.

En resumen, como resultado se obtienen tres simulaciones por cada aula (A1, A2, A3 y A4), en relación a la base climática ARG_Mendoza CCT y los dos husos horarios (ARG_MendozaCCT_UTC-
3; ARG_MendozaCCT_UTC-4). El análisis, en primera instancia, estudia comparativamente los resultados obtenidos en las métricas dinámicas DA (luz natural autónoma) y DAcon (luz natural autónoma continua) ofrecidos por el simulador; y en segunda instancia, analiza el consumo eléctrico anual [KWh] tomando como criterio de evaluación, el control manual $(M c)$ y por fotosensor y dimerizado (Dc) del encendido/apagado de la iluminación artificial en las aulas (A1), (A2), (A3) y (A4) en condiciones (UTC-3) y (UTC-4).

\section{Resultados}

En las siguiente tabla se presentan el relevamiento físico de las tipologías de aulas analizadas en los casos de estudio (a) y (b) (Figura 5), como así también las propiedades fotométricas relevadas in situ, coincidentes con la normativa vigente (Tabla 2).

\section{Configuración de archivos climáticos para los diferentes usos horarios}

A continuación se presentan gráficamente los archivos de climas ARG_MendozaCCT para diferentes husos horario (UTC): ARG_MendozaCCT_UTC-3, ARG_MendozaCCT_UTC-4 y ARG_MendozaCCT_UTC-5 (Tabla 3). Donde el eje $(\mathrm{x})$ representa las semanas del año $[\mathrm{Wk}]$ y el eje (y) las horas del día [Hs].

Tabla 1 - Archivo de ocupación del espacio generado (schoolyearwithholidays.csv)

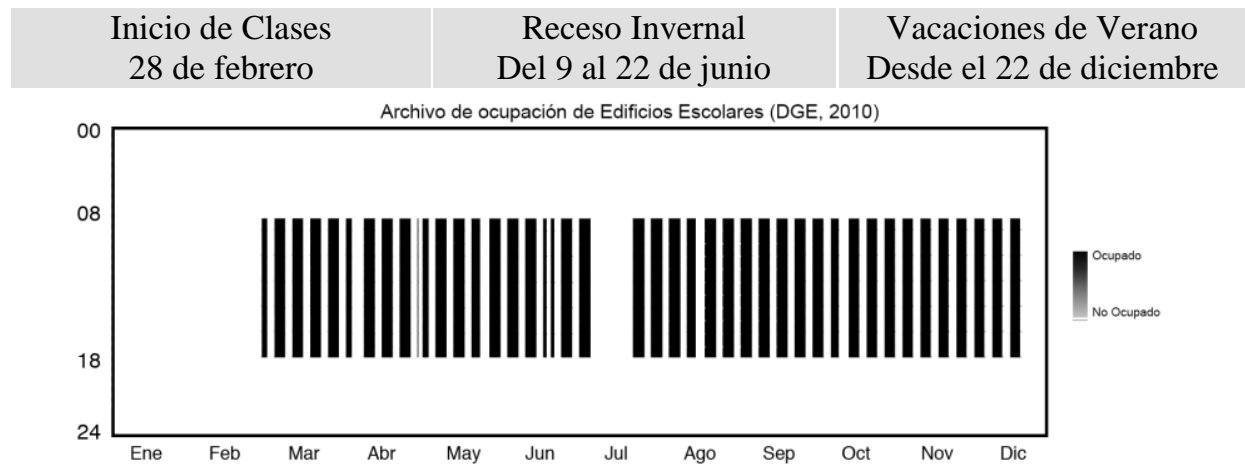

Tabla 2 - Propiedad ópticas de los materiales y características del grillado en el plano útil

\begin{tabular}{l|l}
\hline $\begin{array}{l}\text { Factores de reflexión recomendadas para las superficies interiores } \\
\text { de las aulas (IRAMM AADL J 20 04) coincidentes con las relevadas }\end{array}$ & $\begin{array}{l}\text { Materiales } \\
\text { RADIANCE empleados }\end{array}$ \\
\hline $70-80$ [\% Reflectancia] & beige paint LBNL 2k216 \\
$40-60[\%$ Reflectancia] & beige 2k210 LES091 \\
$10-30$ [\% Reflectancia] & beige-brown 3k313 LES091 \\
T $0.91 \mathrm{Ra} 0.082 \mathrm{Rp} 0.082$ (Pilkington North America) & CLEAR2 LO \\
\hline
\end{tabular}

242 Monteoliva, J. M.; Pattini, A. 
Tabla 3 - Archivos climáticos generados para la ciudad de Mendoza con diferentes (UTC)

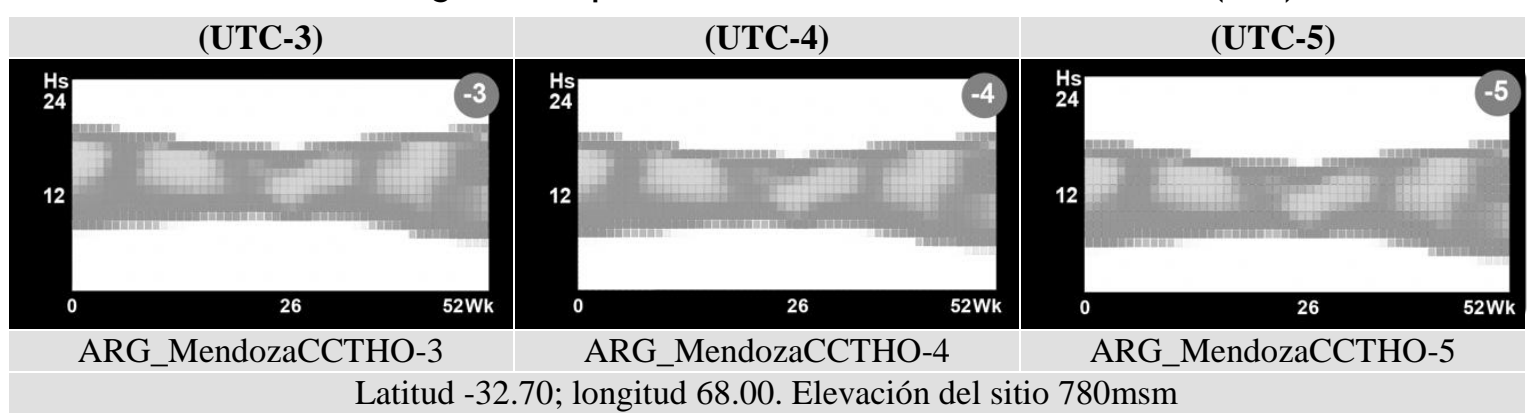

\section{Simulaciones y análisis de métricas de iluminación natural y consumo eléctrico (iluminación)}

\section{Análisis de métricas de iluminación natural: DA500lux y DAcon500lux (Tabla 4)}

En el siguiente apartado se analizan los diferentes espacios seleccionados para los casos $(a)$ y $(b)$ (A1, A2, A3 y A4) en condiciones de UTC-3 y UTC-4. La métricas dinámicas evaluadas, como fue mencionado, son: DA500lux y DAcon500lux. El (DA500lux) obtenido, en el caso de estudio (a), para la condición de UTC-3 fue de $34.7 \%$, 7.4\% y $50.3 \%$; mientras que para UTC-4 de $32.7 \%, 12.8 \%$ y $52.2 \%$, en las aulas (A1), (A2) y (A3) respectivamente. Asimismo la variación promedio ( $\triangle D A 500$ lux $)$ entre UTC-3 y UTC-4 fue del $3 \%$. En relación a la autonomía con ponderación (DAcon500lux) las diferencias promedio encontradas entre UTC-3 y UTC-4 fueron del 13\% $(D S=25.54$ y $M a=4)$. Por otra parte, en el caso de estudio (b) (A4), la autonomía del espacio (DA500lux) obtenida fue del $65.4 \%$ (UTC-3) y $68.2 \%$ (UTC-4). La variación promedio $(\triangle D A 500$ lux $)$ entre ambas condiciones fue del $3 \%$, a igual que en (DAcon500lux).

Al comparar ambas instituciones, el espacio (A4) aula con acondicionamiento bioclimático- presenta la mayor autonomía de los casos analizados con un DA500lux $=65.4 \% \quad($ UTC-3) y $68.2 \%$ (UTC-4); seguido de (A3) - aula tradicional - con un DA500lux $=50.3 \%$ y $52.2 \%$ en condiciones (UTC3) y (UTC-4), respectivamente. La diferencias porcentual en la autonomía de los espacios entre (A3) y (A4) es del $15 \%$ en condiciones UTC-3 y UTC-4. Es decir, el aula con acondicionamiento bioclimática (A4) no sólo aumenta la autonomía en un $15 \%$ actualmente, en relación al mejor acondicionamiento de una aula tradicional (A3); sino que al cambiar la condición a UTC-4 se mantendría esta diferencia.

\section{Consumos eléctricos de iluminación artificial (Tabla 5)}

En el siguiente apartado se analizan los consumos energéticos anuales de la iluminación artificial. Para ello se evalúa predictivamente el consumo energético [KWh], tomando como criterio de evaluación el control manual $(M c)$ y por fotosensor y dimerizado $(D c)$ de la iluminación artificial. A partir de estos criterios se analizan los consumos predictivos para los espacios evaluados en condiciones UTC-3 y UTC-4:

(a) (Mc) Control Manual de la iluminación artificial: en el caso de estudio $(a)$ (A1, A2 y A3) el consumo eléctrico, con control manual de encendido $(M c)$, obtenido para la condición UTC-3 fue de $923.8 \mathrm{KWh}, 909.8 \mathrm{KWh}$ y $884.4 \mathrm{KWh}$; mientras que para UTC-4 de $692.4 \mathrm{KWh}, 683 \mathrm{KWh}$ y 654.8KWh para las aulas (A1), (A2) y (A3) respectivamente. La disminución del consumo (Mc) entre ambas condiciones fue del $25 \%$ $(D S=2.6)$. Por otra parte, en el caso de estudio $(b)$ (A4), el consumo $(M c)$ obtenido fue de $877.6 \mathrm{KWh}$ (UTC-3) y 542.8KWh (UTC-4). La disminución porcentual del consumo eléctrico, entre ambas condiciones, fue del $38 \%$. Al comparar ambas instituciones, el espacio (A4) - aula con acondicionamiento bioclimática- presenta el menor consumo eléctrico de los casos analizados 877.6KWh (UTC-3) y 542.8KWh (UTC-4) -; seguido de (A3) - 884.4KWh (UTC-3) y 654.8KWh (UTC-4). La diferencias porcentual de consumos entre (A3) y (A4) es del 4\% (UTC-3) y del 17\%(UTC-4). Es decir, el aula con acondicionamiento bioclimática (A4) no sólo reduce los consumos en un $4 \%$ actualmente, en relación al mejor acondicionamiento de una aula tradicional (A3); sino que de cambiar a UTC-4 la reducción alcanzaría el 17\%. A continuación, a modo de ejemplo, fueron seleccionados los espacios mejor acondicionados para ser representados gráficamente sus consumos eléctricos anuales $(M c)(D c) ; y$

(b) (Dc) Control con Fotosensor de la iluminación artificial: en el caso de estudio (a) (A1, A2 y A3) 
el consumo eléctrico, con fotosensor y dimerizado (Dc), obtenido para la condición UTC-3 fue de $580 \mathrm{KWh}, 742.2 \mathrm{KWh}$ y $507 \mathrm{KWh}$; mientras que para UTC-4 de 475.8KWh, 557.2KWh y 403.6KWh para las aulas (A1), (A2) y (A3) respectivamente. La disminución porcentual del consumo $(D c)$ entre ambas condiciones fue del $21 \%(D S=3.5)$. En el caso de estudio (b) (A4), el consumo $(D c)$ obtenido fue de $385.2 \mathrm{KWh}$ (UTC-

3) y $270.6 \mathrm{KWh}$ (UTC-4). La disminución porcentual del consumo eléctrico, entre ambas condiciones, fue del 30\%. Al comparar ambas instituciones, el espacio (A4) - aula bioclimática presenta el menor consumo eléctrico de los casos analizados - 385.2KWh (UTC-3) y 270.6KWh (UTC-4) -; seguido de (A3) - 507KWh (UTC-3) y 403.6KWh. La diferencias porcentual de consumos entre (A3) y (A4) es del 24\% (UTC-3) y del $33 \%$ (UTC-4). Es decir, el aula con acondicionamiento bioclimática (A4) no sólo reduce los consumos en un $24 \%$ actualmente, en relación al mejor acondicionamiento de una aula tradicional (A3); sino que de cambiar a UTC-4 la reducción alcanzaría el 33\%.

A continuación se presenta una síntesis de los resultados más representativos, obtenidos en las diferentes tipologías de aulas analizadas (A1), (A2), (A3) y (A4) (Tabla 6).

\section{Conclusiones}

En el desarrollo del trabajo se han analizado comparativamente diferentes acondicionamientos lumínicos, correspondientes a tipologías de aulas más representativas del parque edilicio educativo público tradicional y de acondicionamiento bioclimático de la región. Los resultados permitieron verificar, en tipologías de aulas con sistemas de iluminación natural de aventanamiento bilateral sin acondicionamiento bioclimático caso de estudio (a), tradicionales -, la importancia en del factor orientación $(\mathrm{N}-\mathrm{S})$ en la región y la adecuada relación entre la superficie vidriada y el área del piso - establecido por la normativa nacional vigente IRAM AADL 20 02. Esto se evidencia en el espacio ( $A 3)$ con una autonomía de iluminación natural DA500lux $=50 \%$ para ambas condiciones; siendo ésta, la mayor autonomía registrada en el caso (a). Sin embargo, cabe destacar que esta tipología tradicional ocasionalmente presenta similitudes a un acondicionamiento bioclimático. Éste, está dado por un "sistema de redirección de la radiación solar"; generado por el techo del pasillo exterior (N) y su recubrimiento con un material semiespecular (membrana).

\section{Tabla 4 - DA500lux de los diferentes espacios en condiciones (UTC-3) (UTC-4)}

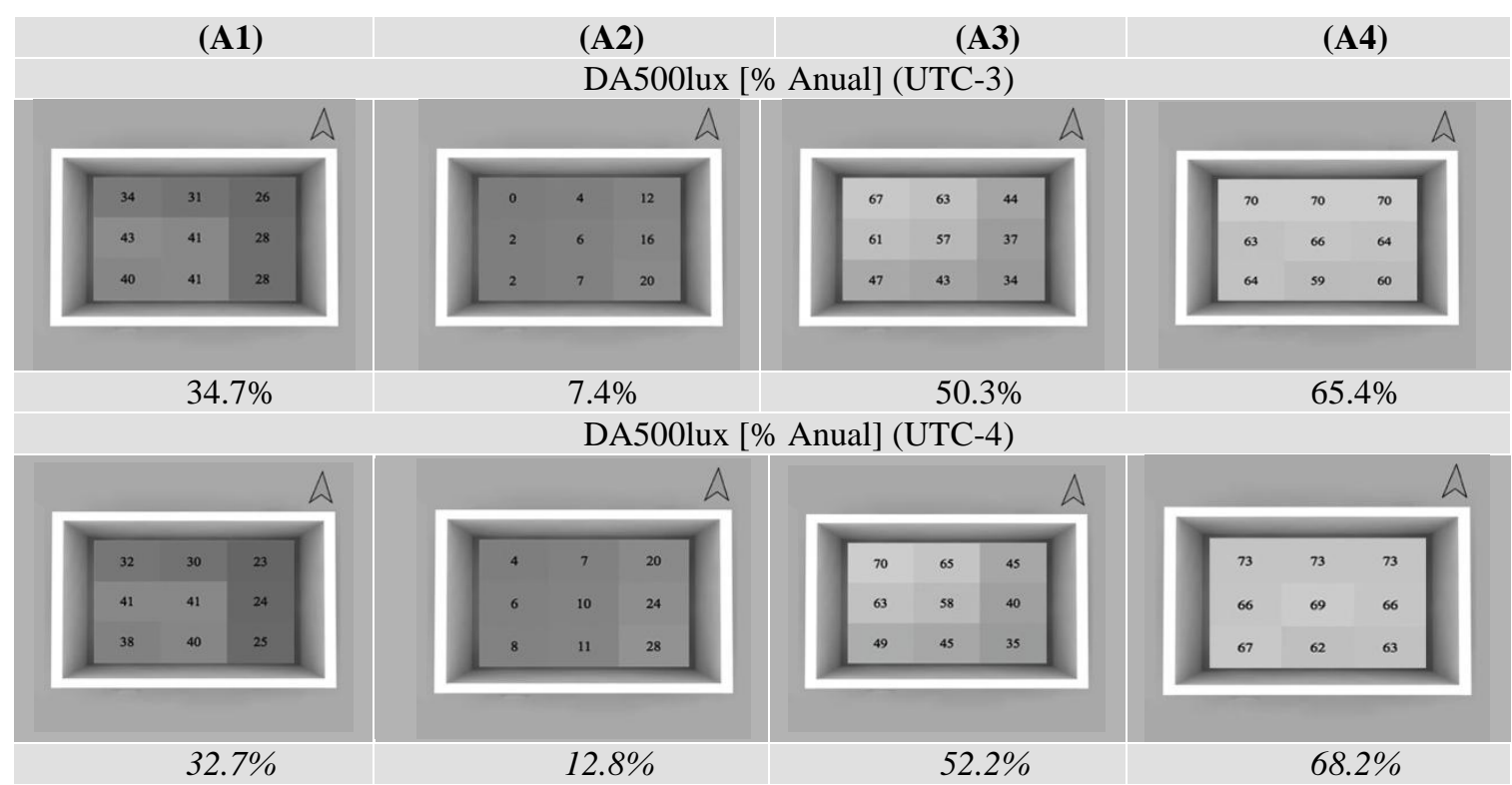

244 Monteoliva, J. M.; Pattini, A. 
Tabla 5 - Evaluación predictiva anual de encendido/apagado de la iluminación artificial

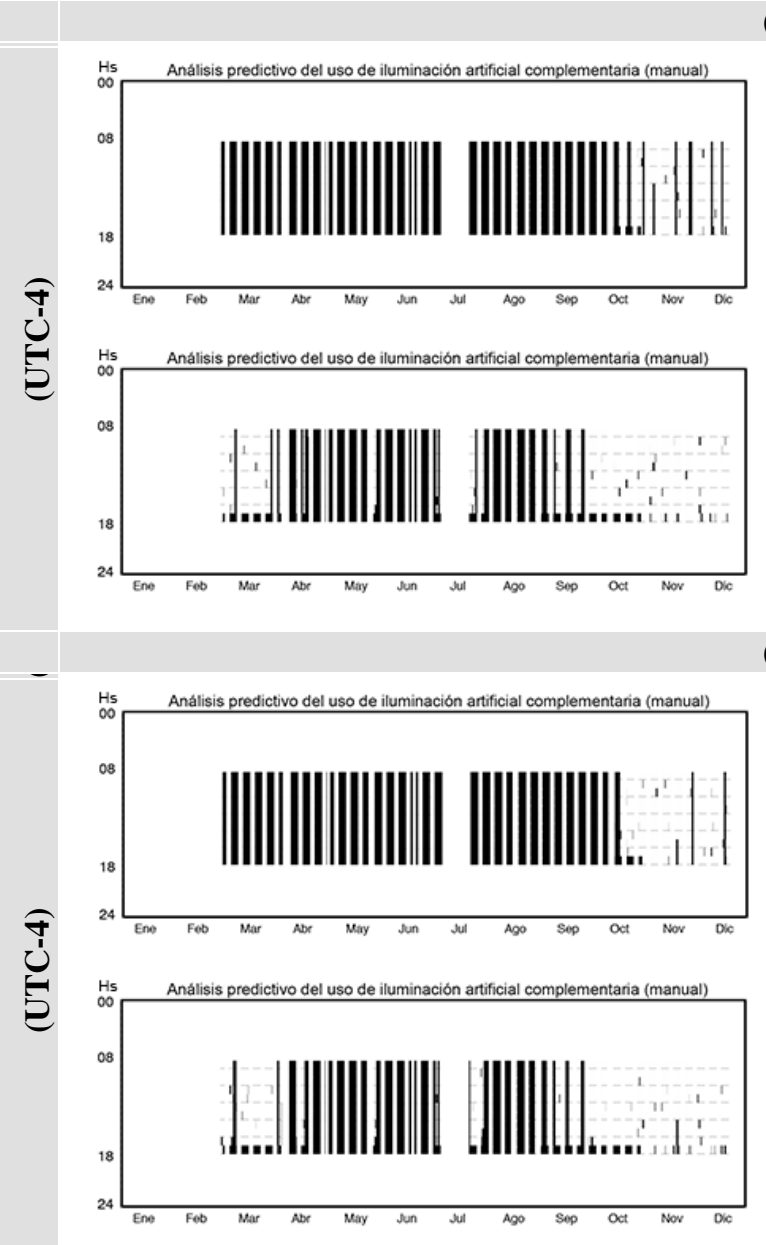

Control Manual Encendido/Apagado
(A3)

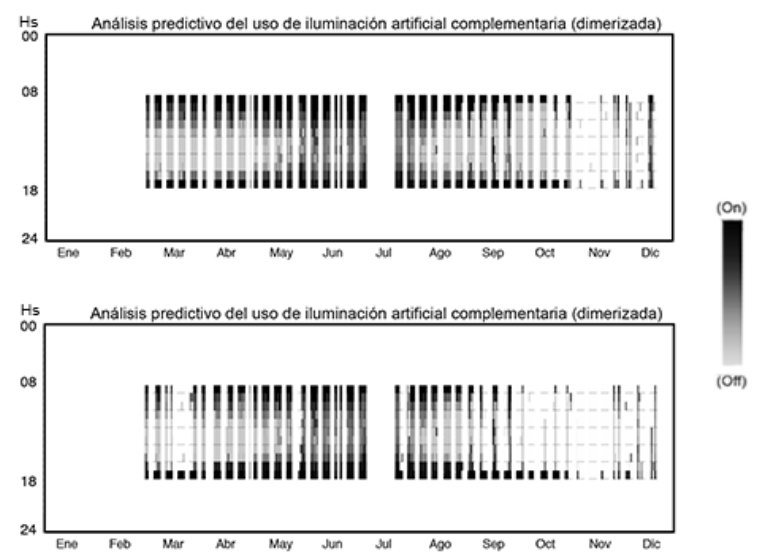

(A4)

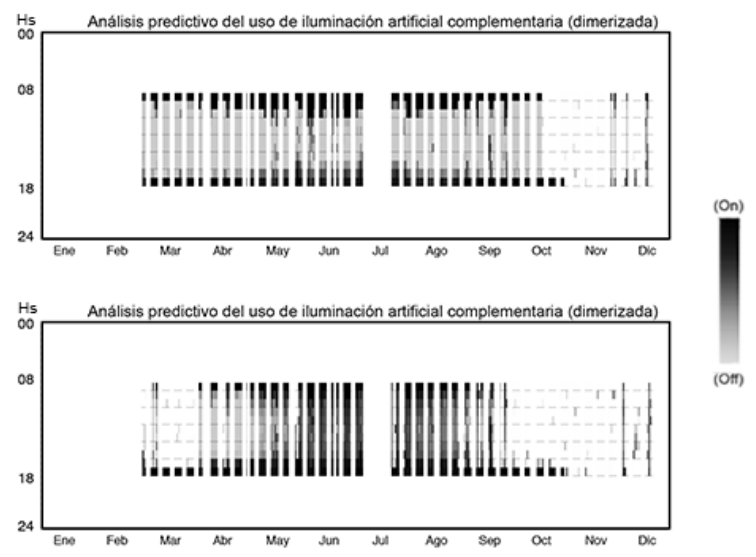

Control por Dimerizado Encendido/Apagado

Tabla 6 - Síntesis de resultados en condiciones (UTC-3) (UTC-4)

\begin{tabular}{c|c|c|c|c|c|c}
\hline & \multicolumn{3}{|c|}{ UTC-3 } & \multicolumn{3}{c}{ UTC-4 } \\
\cline { 2 - 7 } & DA500lux & Mc & Dc & DA500lux & Mc & Dc \\
\hline A1 & $34.7 \%$ & $923.8 \mathrm{KWh}$ & $580 \mathrm{KWh}$ & $7.4 \%$ & $692.4 \mathrm{KWh}$ & $475.8 \mathrm{KWh}$ \\
A2 & $32.7 \%$ & $909.8 \mathrm{KWh}$ & $742.2 \mathrm{KWh}$ & $12.8 \%$ & $683 \mathrm{KWh}$ & $557.2 \mathrm{KWh}$ \\
\hline A3 & $50.3 \%$ & $454.9 \mathrm{KWh}$ & $290.0 \mathrm{KWh}$ & $52.2 \%$ & $341.5 \mathrm{KWh}$ & $237.9 \mathrm{KWh}$ \\
A4 & $65.4 \%$ & $438.9 \mathrm{KWh}$ & $192.6 \mathrm{Wh}$ & $68.2 \%$ & $271.4 \mathrm{KWh}$ & $135.3 \mathrm{KWh}$ \\
\hline
\end{tabular}

Nota: *Espacios de mejor acondicionamiento lumínico a lo largo del año en caso de estudios (a) y (b).

Al comparar estos resultados de (A3) con (A4) espacios de acondicionamiento bioclimático con sistemas de iluminación natural de aventanamiento unilateral (N) y cenital -; se observa un aumento de DA500lux del 15\%. Es decir, (A4) presenta un DA500lux $=65.4 \%($ UTC-3) y del 68.2\% (UTC-4). Esto claramente evidencia la eficiencia del acondicionamiento bioclimático y el uso de diseños y estrategias aplicadas de iluminación natural, en una región suficientemente "energética" en términos de eficacia luminosa. Asimismo, en relación al consumo eléctrico, como era de esperar, se establece una relación lineal con la autonomía de la iluminación natural de los espacios; es decir, a mayor autonomía menor consumo. Cabe destacar, en el estudio predictivo del consumo eléctrico por iluminación artificial, el impacto de nuevas tecnologías en el control automático por fotosensor y dimerizado $(D m)$ de encendido/apagado. Éste a comparación del sistema de control manual $(M c)$ genera un ahorro energético del 30\% (120KWh).

En relación al análisis de la influencia del huso horario, se observa claramente una mayor 
disponibilidad del recurso -iluminación natural - a partir de la condición UTC-4, alcanzando variaciones en la autonomía de los espacios por iluminación natural del $3 \%$ en los casos analizados. Sin embargo, cabe destacar que durante el período de mayor disponibilidad de recurso (solsticio de verano), los espacios se encuentran sin uso (Tabla 1). En el caso de ser estos edificios ocupados a lo largo del año por actividades como talleres de la tercera edad o actividades académicas inter-semestrales, el ahorro en el consumo eléctrico de iluminación artificial complementaria alcanzaría los $140 \mathrm{KWh}$. Por otro lado, como fue mencionado, Argentina está ubicada en su totalidad en las franjas de husos horario UTC -4 $\left(-60^{\circ}\right)$ y UTC $-5\left(-75^{\circ}\right)$ (Figura 1$)$; sin embargo, el horario actualmente empleado es el de la franja UTC -3 (-45 ) alejado de la hora solar. Esto implica la importancia de aclarar y consensuar el impacto de un huso horario adecuado a nuestro país, incluyendo en el análisis la decisión del uso de uno o dos horarios para el territorio nacional; como así también la adopción del cambio de hora de verano. La adopción del cambio horario de verano, es una medida incompleta para producir un ahorro de energía eléctrica, más aun si ésta parte de un huso horario inadecuado. El cual no sólo afecta energéticamente sino en la salud y estados de ánimo de sus ciudadanos (KIRSCHBAUM; TONELLO, 2010).

Los resultados obtenidos a través de este análisis son claramente alentadores. A partir de éstos, se espera contribuir a una mayor concientización sobre la factibilidad y beneficios de los edificios escolares energéticamente eficientes; como así también en la importancia de la iluminación natural como factor influyente desde diferentes perspectivas como la energía, salud y confort. De ocurrir, será posible profundizar en diseños y tecnologías de sistemas de iluminación natural aplicados de forma masiva en el futuro; generando un mejor aprovechamiento y control de la luz natural dentro de las instituciones educativas. Un gran paso hacia el objetivo de la sustentabilidad ambiental.

\section{Referencias}

ANDERSEN M. Pioneering Daylighting Design Tool to combine Architectural Form with Advanced Technology: The LightSolve Project. Massachusetts: Building Technology Program, 2007.
ANTROP. I.; ROEYERS, H.; DE BAECKE, L. Effects of Time of Dayon Classroom Behavior in Children with ADHD. School Psychology International, v. 26, n. 1, p.29-34, 2005.

BODART, M.; DE HERDE, A. Global Energy Savings in Office Buildings By Use of Daylighting. Energy and Buildings, v. 34, n. 5, p. 421-429, 2002.

BOYCE, P.; HUNTER, C.; HOWLETT, O. The Benefits of Daylight through Windows. New York: Lighting Research Center, Rensselaer Polytechnic Institute, 2003.

CANTÓN, A.; DE ROSA, C.; KASPERIDUS, H. Sustentabilidad del Bosque Urbano en el Área Metropolitana de la Ciudad de Mendoza: análisis y diagnóstico de la condición de las arboledas. Avances en Energías Renovables y Medio Ambiente, v. 7, n. 1, p. 29-34, 2003.

CENTRO DE EDUCACIÓN EN APOYOA LA PRODUCCIÓN Y AL MEDIO AMBIENTE. A.C. CEP México, Editorial Parras. Los Ambientes Educativos. ¿Generadores de Capital Humano? Revista Debate en Educación de Adultos, v. 7, p. 15-18, 1997.

CÓRICA, L. Comportamiento de la Luz Natural en Entornos Urbanos Representativos del Modelo Oasis en Regiones Áridas. Caso de Estudio: ciudad de Mendoza. Tucumán, 2009. Tesis (Doctorado) - Departamento de Luminotecnia, Luz y Visión Herberto Büller, Universidad Nacional de Tucumán, Tucumán, 2009.

DUNN, R. et al. Light Up Their Lives: a research on the effects of lighting on children's achievement and behavior. The Reading Teacher, v. 38, n. 19, p. 863-869, 1985.

EVANS, G. W.; FERGUSON, K. T. Built Environment and Mental Health. In: NRIAGU, Jerome O. (Ed.). Encyclopedia of Environmental Health. Burlington: Elsevier, 2011. p. 446-449.

FONTOYNONT, M. Daylight Performance of Buildings. Lyon: ENTPE, 1999.

GALASIU, A. D.; VEITCH, J. A. Occupant Preferences and Satisfaction With the Luminous Environment and Control Systems in Daylit Offices: a literature review. Energy and Buildings, v. 38, n. 7, p. 728-742, 2006.

HESCHONG, L. Daylighting in Schools: an Investigation into the Relationship Between Daylighting and Human Performance. Fair Oaks: Heschong Mahone Group to Pacific Gas and Electric, 1999. 
INSTITUTO ARGENTINO DE

NORMALIZACIÓN Y CERTIFICACIÓN.

Asociación Argentina de Luminotecnia. IRAM-

AADL J20-04: iluminación en escuelas:

características. Bahia Blanca, 1974.

INSTITUTO ARGENTINO DE

NORMALIZACIÓN Y CERTIFICACIÓN.

Asociación Argentina de Luminotecnia. IRAM-

AADL J20-02: iluminación natural en edificios: condiciones generales y requisitos especiales.

Bahia Blanca, 1970.

ISON, M. S.; PATTINI, A. E. Eficacia Atencional en Niños y Optimización del Acondicionamiento Lumínico en Aulas. Investigación en Ciencias del Comportamiento. Avances Iberoamericanos.

Buenos Aires: CIIPME- CONICET, 2009. p. 8599.

JAKUBIEC, J. A.; REINHART, C. F. The

'Adaptive Zone': a concept for assessing glare throughout daylit spaces. Lighting Research \& Technology, v. 44, n. 2, p. 149-70, 2012.

JARVIS, D.; DONN, M. Comparison of Computer and Model Simulations of a Daylit Interior With Reality. In: INTERNATIONAL IBPSA CONFERENCE, 5., Prag, 1997. Proceedings... Prag, 1997.

KIRSCHBAUM, C.; TONELLO, G. Daylight Use and Local Time Shift Assessment: an exploratory study in Argentina. In: LIGHTING QUALITY AND ENERGY EFFICIENC, Vienna, 2010. Proceedings... Vienna, 2010.

LESESNE, C. A.; VISSER, S. N.; WHITE, C. P. Attention-Deficit/Hyperactivity Disorder in School-Aged Children: association with maternal mental health and use of health care resources. Pediatrics, v. 111, n. supplement 1, p. 1232-1237, may 2003.

LI, D. H. W. et al. Lighting and Energy Performance of Solar Film Coating in AirConditioned Cellular Offices. Renewable Energy, v. 29, n. 6, p. 921-937, 2004.

MAXWELL, E. L. Un Modelo Cuasi-Física Para la Conversión de Hora Mundial Horizontal a Direct Insolación Normal. Golden, CO: Solar Energy Research Institute, 1987. Informe Técnico $\mathrm{N}^{\circ}$ SERI/TR-215-3087.

MCNEEL, R. et al. Rhinoceros Version 4.0. 2010. Service Release.

MINISTERIO DE EDUCACIÓN DE LA REPÚBLICA ARGENTINA. Dirección de Infraestructura. Criterios y Normativa Básicas de Arquitectura Escolar. Oct. 1998.
MINISTERIO DE EDUCACIÓN DE LA REPÚBLICA ARGENTINA. Dirección General de Escuelas de la Provincia de Mendoza. Calendario Escolar para el Ciclo Lectivo 2010. Dic. 2009.

MINISTERIO DE TRABAJO, EMPLEO Y SEGURIDAD SOCIAL. Superintendencia de Riesgos de Trabajo. Protocolo de medición de iluminación en el ambiente laboral. Enero 2012.

MITCHELL, J. et al. Escuela Marcelino Blanco: un edificio energéticamente eficiente en el este de Mendoza. Avances en Energías Renovables y Medio Ambiente, v. 3, n. 1, 1999.

MONTEOLIVA, J. M.; VILLALBA, A.; PATTINI, A. Impacto de la Utilización de Bases Climáticas Regionales en la Simulación de Alta Precisión de Iluminación Natural. Avances en Energías Renovables y Medio Ambiente, v. 16, 2012.

MONTEOLIVA, J. M. Influencia del Diseño de la Iluminación en Aulas Sobre la Eficacia Atencional en Niños: impacto de la luz natural. Mendoza: CONICET, 2010. Beca Interna Tipo II.

OKURA, S.; HESCHONG, L.; WRIGHT, R. Skylighting and Retail Sales. ACEEE Summer Study on Energy Efficiency in Buildings, v. 8, p. 245-56, 2000.

PATTINI, A. La Luz Natural en las Escuelas: aprovechamiento y control de la luz solar en aulas. Buenos Aires: Dunken, 2009.

PEREZ, R. et al. Modeling Daylight Availability and Irradiance Components From Direct and Global Irradiance. Solar Energy, v. 44, n. 5, p. 271-289, 1990.

PEREZ, R.; SEALS, R.; MICHALSKY, J. AllWeather Model for Sky Luminance Distribution: preliminary configuration and validation. Solar Energy, v. 50, n. 3, p. 235-245, 1993.

PHILLIPS, R. W. Educational Facility Age and the Academic Achievement of Upper Elementary School Students. Georgia, 1997. Thesis (Doctoral Dissertation) - University of Georgia, Georgia, 1997.

REA, M. S. Light: much more than vision. In: International Lighting Research Symposium, 5., Palo Alto, 2002. Proceedings... Palo Alto, 2002.

REINHART, C. F. Tutorial on the Use of DAYSIM Simulations for Sustainable Design. Ottawa: Institute for Research in Construction, National Research Council Canada, 2006. 
REINHART, C.; MARDALJEVIC, J.; ROGERS, Z. Dynamic Daylight Performance Metrics for Sustainable Building Design. Leukos, v. 3, n. 1, p. 1-20, 2006.

REINHART, C. F. Lightswitch 2002: a model for manual and automated control of electric lighting and blinds. Solar Energy, v. 77, n. 1, p. 15-28, 2004.

REINHART, C. F. et al. DIVA for Rhino

Version 2.0. 2011. Disponíble en:

<http://www.diva-for-rhino.com/>. Acceso em: 25 nov. 2013.

ROGERS, Z. Daylighting Metric Development Using Daylight Autonomy Calculations in the Sensor Placement Optimization Tool. Boulder, Colorado: Architectural Energy Corporation. 2006.

TAYLOR, A.; GOUSIE, G. The Ecology of Learning Environments For Children. CEFPI Journal, v. 26, n. 4, p. 23-28, 1988.

TONELLO G. Efectos no Visuales de la Luz: una puesta al día. Revista Luminotecnia, n. 56, 1998.

VEITCH, J. A.; NEWSHAM, G. R. Lighting Quality and Energy-Efficiency Effects on Task Performance, Mood, Health, Satisfaction and Comfort. JIES, v. 27, n. 1, p. 107-129, winter 1998.

VEITCH, J. A.; GIFFORD, R.; HINE, D. W. Demand Characteristics and Full Spectrum Lighting Effects on Performance and Mood. Journal of Environmental Psychology, v. 11, p. 97-95, 1991.
WARD, L. G.; SHAKESPEARE, R. Rendering With Radiance: the art and science of lighting visualization. San Francisco: Morgan Kaufmann Publishers, 1998.

WEBB, A. R. Considerations For Lighting in the Built Environment: non-visual effects of light. Energy and Buildings, v. 38, n. 7, p. 721-727, 2006.

WIENOLD, J.; CHRISTOFFERSEN, J.

Evaluation Methods and Development of a New Glare Prediction Model For Daylight

Environments With the Use of CCD Cameras.

Energy and Buildings, v. 38, n. 7, p. 743-757, 2006.

WU, W.; NG, E. A Review of the Development of Daylighting in Schools. Lighting Research and Technology, v. 35, n. 2, p. 111-125, 2003.

\section{Agradecimientos}

Este trabajo fue financiado con los proyectos:

- PICTO ENARGAS 2009-0133: Desarrollo y Estudio de Comportamiento Energético de Precisión de Carpinterías Exteriores y Elementos de Control Solar de Bajo Costo. FONCYT, Agencia Nacional de Promoción Científica y Tecnológica, Argentina; y

- PIP 0133 CONICET: Barreras que Obstaculizan el Uso De Iluminación Natural en el Hábitat Construido. Identificación y Propuestas de Superación en Climas Soleados.

\title{
Juan Manuel Monteoliva
}

Laboratorio de Ambiente Humano y Vivienda | Av. Ruiz Leal, s/n, Parque Gral, INCIHUSA - CCT CONICET | Tel.: +(54) 261 524-4322 |

San Martín - Mendoza - Argentina | E-mail: jmonteoliva@mendoza-conicet.gob.ar

\section{Andrea Pattini}

Laboratorio de Ambiente Humano y Vivienda | Mendoza - Argentina | E-mail: apattini@mendoza-conicet.gob.ar

\author{
Revista Ambiente Construído \\ Associação Nacional de Tecnologia do Ambiente Construído \\ Av. Osvaldo Aranha, $99-3^{\circ}$ andar, Centro \\ Porto Alegre - RS - Brasil \\ CEP $90035-190$ \\ Telefone: +55 (51) 3308-4084 \\ Fax: +55 (51) 3308-4054 \\ www.seer.ufrgs.br/ambienteconstruido \\ E-mail: ambienteconstruido@ufrgs.br
}

248 Monteoliva, J. M.; Pattini, A. 